\title{
Flexible Cortical Control of Task-Specific Muscle Synergies
}

\author{
Kianoush Nazarpour, Amy Barnard, and Andrew Jackson \\ Institute of Neuroscience, Newcastle University, Newcastle-upon-Tyne NE2 4HH, United Kingdom
}

Correlation structure in the activity of muscles across movements is often interpreted as evidence for low-level, hardwired constraints on upper-limb function. However, muscle synergies may also emerge from optimal strategies to achieve high-level task goals within a redundant control space. To distinguish these contrasting interpretations, we examined the structure of muscle variability during operation of a myoelectric interface in which task constraints were dissociated from natural limb biomechanics. We found that, with practice, human subjects learned to shape patterns of covariation between arbitrary pairs of hand and forearm muscles appropriately for elliptical targets whose orientation varied on a trial-by-trial basis. Thus, despite arriving at the same average location in the effector space, performance was improved by buffering variability into those dimensions that least impacted task success. Task modulation of betafrequency intermuscular coherence indicated that differential recruitment of divergent corticospinal pathways contributed to positive correlations among muscles. However, this feedforward mechanism could not account for negative correlations observed in the presence of visual feedback. A second experiment revealed the development of fast, target-dependent visual responses consistent with "minimum intervention" control correcting predominantly task-relevant errors. Together, these mechanisms contribute to the dynamic emergence of task-specific muscle synergies appropriate for a wide range of abstract task goals.

\section{Introduction}

Redundancy is a ubiquitous feature of the musculoskeletal system (Bernstein, 1967). Kinematics and muscle patterns are in general underdetermined by specific movement goals, so understanding what additional constraints govern our actions is a central theme of motor systems research. Two principal approaches have emerged, distinguished by whether constraints are imposed in the low-level implementation of the motor system or through high-level optimization of behavior.

The former proposes that hardwired neural circuitry constrains movements such that seemingly diverse actions are constructed from a small number of motor primitives, perhaps organized within spinal modules (Bizzi et al., 1995, 2000; Drew et al., 2008; Hart and Giszter, 2010; Takei and Seki, 2010; Dominici et al., 2011). This reduction in degrees of freedom imposes correlations between muscles across different movements, sometimes referred to as muscle synergies (d'Avella et al., 2003) or M-modes (Krishnamoorthy et al., 2003).

A contrasting view emerges from studying repetitions of the same movement. In any redundant task, we can define an uncontrolled manifold in effector space along which deviations do not affect task success (Scholz and Schöner, 1999; Latash et al., 2001). Latash et al. (2002) define a synergy as the shaping of trial-to-trial movement variability along this manifold. In computational terms, a minimum

\footnotetext{
Received Oct. 31, 2011; revised June 13, 2012; accepted June 19, 2012.

Author contributions: K.N. and A.J. designed research; K.N. and A.B. performed research; K.N. contributed unpublished reagents/analytic tools; K.N. analyzed data; K.N. and A.J. wrote the paper.

This work was supported by Medical Research Council Grant G0802195 and Wellcome Trust Grant 086561.

The authors declare no competing financial interests.

This article is freely available online through the J Neurosci Open Choice option.

Correspondence should be addressed to Kianoush Nazarpour, Institute of Neuroscience, Newcastle University, Newcastle-upon-Tyne NE2 4HH, UK. E-mail: k.nazarpour@ncl.ac.uk.

DOI:10.1523/JNEUROSCI.5481-11.2012

Copyright $\odot 2012$ the authors $\quad 0270-6474 / 12 / 3212349-12 \$ 15.00 / 0$
}

intervention strategy that controls only task-relevant degrees of freedom is optimal if there is a cost associated with correcting variability in the uncontrolled manifold (Todorov and Jordan, 2002; Scott, 2004; Diedrichsen et al., 2010). Importantly, in this interpretation, synergies are not imposed by neural architecture but emerge from high-level control policies to achieve movement goals optimally in the presence of biomechanical redundancy.

Since any movement of a limb is influenced by the same biomechanics, we might expect these optimal synergies to be broadly conserved across natural behaviors (Chhabra and Jacobs, 2006). This has led some to propose that hardwired muscle synergies evolved as computational shortcuts approximating optimal strategies (Todorov et al., 2005; Berniker et al., 2009; Tresch and Jarc, 2009; Giszter et al., 2010). In practice, it is difficult to distinguish low- and high-level interpretations of synergies by studying movements within the ethological repertoire since the synergies predicted by optimal control theory could always be explained by appropriate hardwired circuits (Valero-Cuevas et al., 2009). However, if correlation structure reflects a flexible strategy to optimize behavior, then a strong prediction is that appropriate muscle synergies should emerge in any abstract, redundant task. Therefore, we used a myoelectric interface (Radhakrishnan et al., 2008) controlled directly by electromyogram (EMG) signals to study movements dissociated from the physical limb. To reveal the influence of high-level constraints on correlation structure, we quantified trial-to-trial variability as subjects moved to the same average location in muscle space, while varying the task axis most relevant to success. We found that rapid adaptation of both feedforward drive via divergent corticospinal projections and visuomotor feedback loops contribute to form dynamic, taskdependent muscle synergies. These results reveal how the human motor cortex can transcend hardwired constraints and optimize hand function for the demands of abstract task goals. 
A

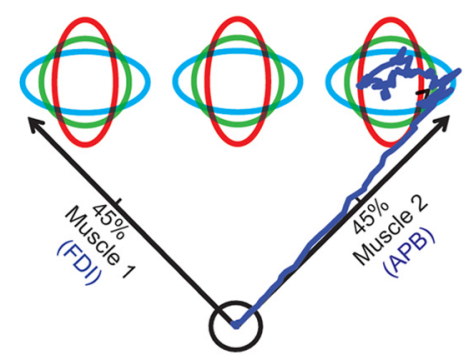

B

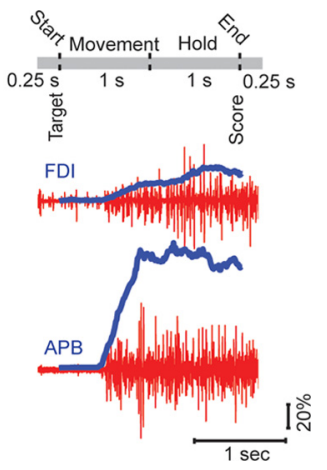

Figure 1. Task design. $\boldsymbol{A}$, Muscle pairs controlled the cursor along axes diagonal to taskrelevant and -irrelevant dimensions. $\boldsymbol{B}$, Each trial was divided into $1 \mathrm{~s}$ movement and hold periods. Control signals (blue traces) were computed at $75 \mathrm{~Hz}$ by rectifying and smoothing the preceding $500 \mathrm{~ms}$ of EMG (red traces) to determine the instantaneous position of the myoelectric cursor.

\section{Materials and Methods}

\section{Participants}

In total, 36 right-handed subjects participated in this study. No subject took part in more than one experiment and all were naive to the experimental setup and objectives. They were free of any history of neurological or motor disorders and gave informed consent. The study was approved by the local ethics committee at Newcastle University.

\section{Experimental setup}

Subjects controlled a myoelectric cursor by making isometric contractions of right upper-limb muscles, as described by Radhakrishnan et al. (2008). The hand was restrained in an open, pronated posture inside a glove fixed to a horizontal board and the forearm was strapped to the armrest of the chair. The cursor and targets were displayed on a computer screen positioned in front of the subject. Surface EMG from hand and forearm muscles was amplified (gain, $1 \mathrm{~K}-10 \mathrm{~K}$ ) and high-pass filtered at $30 \mathrm{~Hz}$ (Neurolog NL824/820; Digitimer) before sampling at $5 \mathrm{kHz}$ (PCI6071E; National Instruments). At the start of the experiment, subjects were informed of the general task design and the hand movements that recruited each recorded muscle. They were then instructed to contract each muscle at a level that they would comfortably be able to repeat many times without fatigue. Subsequently, myoelectric control signals were normalized by this level (100\% of comfortable contraction), which typically corresponded to between 10 and $20 \%$ of maximum voluntary contraction (measured at the end of the session). Control signals were computed every 13 ms by smoothing (with a rectangular window) the preceding $500 \mathrm{~ms}$ of rectified EMG. During the task, the control signals from a pair of muscles determined the instantaneous position of the myoelectric cursor along orthogonal axes $\left(m_{1}, m_{2}\right)$ that were diagonal to screen coordinates $(x, y)$ (Fig. $1 A)$ as follows:

$$
\begin{aligned}
& m_{1}(t)=\alpha_{1} \cdot \sum_{\delta=0}^{500 \mathrm{~ms}}\left|\mathrm{EMG}_{1}(t-\delta)\right|, \\
& m_{2}(t)=\alpha_{2} \cdot \sum_{\delta=0}^{500 \mathrm{~ms}}\left|\mathrm{EMG}_{2}(t-\delta)\right| \\
& x(t)=\frac{1}{\sqrt{2}}\left[m_{2}(t)-m_{1}(t)\right], \quad y(t)=\frac{1}{\sqrt{2}}\left[m_{1}(t)+m_{2}(t)\right],
\end{aligned}
$$

where $\left|\mathrm{EMG}_{j}(t)\right|$ denotes the rectified activity of muscle $j$ at time $t$. The coefficients $\alpha_{1}$ and $\alpha_{2}$ normalize the control signals by the comfortable contraction level.

Subjects initiated a trial by relaxing the hand to bring the cursor to a starting zone and remaining there for $250 \mathrm{~ms}$ after which a target appeared. The remainder of the trial was divided into two fixed periods lasting $1 \mathrm{~s} \mathrm{each,} \mathrm{designated} \mathrm{movement} \mathrm{and} \mathrm{hold} \mathrm{periods} \mathrm{(Fig.} 1 B$ ). Auditory tones cued the start of the movement and hold periods. At the end of the trial, subjects received a score reflecting the proportion of the hold

period that the cursor was inside the target and were instructed to maximize their score in each trial. Note that, since the trial periods were of fixed duration, the cursor was not necessarily in target at the end of the "movement" period and could move in and out of target during the hold period (although this resulted in a lower score). Targets were presented pseudorandomly at three locations (Fig. 1A); the central target required $\sim 45 \%$ of comfortable contraction in both muscles.

\section{Experiment 1: structured variability in hand muscles}

Sixteen subjects (eight females; mean age, $28 \pm 5$ years) participated in Experiment 1 which was designed to examine whether appropriate structure in muscle variability would arise from high-level, abstract task constraints. We used elliptical target shapes to impose a task-relevant dimension within the cursor space. Ellipses were oriented with the long axis either vertical or horizontal on the screen such that greater variability could be tolerated in the dimension of either positive or negative EMG covariance, respectively. The width of the major and minor axes of ellipses corresponded to $\sim 23$ and $11.5 \%$ of comfortable contraction, respectively. Performance with elliptical targets was compared with circular targets of an equal area. We describe the short and long axes of elliptical targets as the task-relevant and -irrelevant dimensions, although it should be noted that both are constrained by the targets and this terminology reflects their relative importance to task success.

In Experiment 1, EMG signals were recorded concurrently from five hand and forearm muscles: abductor policis brevis (APB) (abducts thumb), first dorsal interosseus (FDI) (abducts the index finger), abductor digiti minimi (ADM) (abducts little finger), extensor carpi radialis (ECR) (extends the wrist), and flexor carpi radialis (FCR) (flexes the wrist). Four pairs of muscles were used to control the task: FDI-ADM (biomechanically independent), FDI-APB (synergistic in precision grip), ECR-FCR (antagonists), and ECR-APB (biomechanically independent). In each condition, we call the muscles that control the task the cursor-related muscles (CRMs), in contrast to the remaining three noncursor-related muscles (NCRMs). The order of CRM pairs was counterbalanced across subjects. For each CRM pair, subjects performed four consecutive blocks of 99 trials (11 pseudorandom presentations of the three target shapes at three locations). During 2 of 11 presentations of each target, the subjects received no visual feedback of cursor position during the movement and hold periods, but still heard the auditory cues. In these trials, they were shown the instantaneous cursor position at the end of the hold period and received their score at the end of the trial. Subjects did not know whether visual feedback would be available until after the movement period was initiated. A rest period of maximum 2 min was allowed after recording from each pair.

\section{Experiment 2: response to visual perturbation}

Eight subjects (four females; mean age, $24 \pm 4$ years) participated in this experiment, which explored the influence of high-level, abstract task constraints on visuomotor feedback responses to sudden perturbation of the cursor position. Subjects performed eight consecutive blocks of 96 trials with visual feedback (vertical and horizontal elliptical targets shapes only; in 80 of 96 trials, the target was in the central position). EMG signals were recorded only from muscles APB and ADM (biomechanically independent) with which subjects controlled cursor position. In one-half of the trials (always with the target in the central position), the cursor was shifted along the axis of one of the controlling muscles in the positive direction. The perturbation occurred $300 \mathrm{~ms}$ into the hold period and persisted until the end of that trial. The magnitude of the shift was $16.6 \%$ of the comfortable contraction level. As before, subjects were instructed to maximize the score presented at the end of each trial, which was consistent with the visual position of the cursor as displayed to the subject (rather than the true level of muscle activity in perturbation trials).

\section{Experiment 3: response to peripheral nerve stimulation}

Twelve subjects (six females; mean age, $22 \pm 4$ years) participated in this extension to Experiment 2, incorporating peripheral nerve stimulation as well as visual perturbation. Subjects performed 16 blocks of 96 trials. EMG signals were recorded from muscles APB and ADM only. In this experiment, in one-half of the trials a perturbation was delivered $300 \mathrm{~ms}$ into the $1 \mathrm{~s}$ hold period; perturbations were either visual (as in Experi- 
ment 2) or somatosensory. Somatosensory perturbations were produced by stimulating either the median nerve at the wrist (a mixed nerve relaying both efferent commands to the thumb and afferent inputs from radial parts of the hand) or the cutaneous nerve of the little finger (which comprises only sensory fibers). A single monophasic stimulus $(0.2 \mathrm{~ms}$ pulse width) was delivered per trial using a DS7A (Digitimer) stimulator, with equal probability to either median or cutaneous nerve. Median nerve stimuli were delivered through adhesive surface electrodes (cathode distal) at an intensity of 1.2 times resting motor threshold for producing an M-wave in APB. Cutaneous nerve stimuli were delivered through ring electrodes placed around the middle and proximal phalanxes of the little finger (cathode proximal) at 2.5 times the threshold for sensory perception.

In electrical stimulation trials, visual feedback was withheld after the end of the movement period so that visuomotor feedback did not override somatosensory corrections. In addition, because stimulation artifact disrupts the myoelectric cursor, subjects did not receive a score at the end of stimulation trials. Therefore, to ensure subjects continued trying to remain inside the target even when they could not see the cursor, we included some trials with neither visual feedback nor stimulation after which the subjects received a score.

In assessing responses to visual perturbation, subject data from Experiment 2 and Experiment 3 were analyzed together (20 subjects). When measuring responses to peripheral nerve stimulation, data from one subject were discarded because of large electrical stimulation artifacts on the EMGs.

A MATLAB R14-based graphical user interface linked to Cogent2000 (Functional Imaging Laboratory, Wellcome Department of Imaging Neuroscience, University College London, London, UK) was developed to implement these experiments.

\section{Data analysis}

Performance scores. Learning was quantified off-line using the same performance metric that was displayed to the subject during the task (i.e., percentage of hold period time that the cursor was within target). Scores for particular target conditions were averaged across all trials within a block, and we used regression analysis across blocks to assess performance improvements (relative to block 1) with training.

Index of covariation. We used the variance of the instantaneous cursor position across different trials to quantify correlation structure of muscle activity. We chose to analyze cursor position (rather than smoothing the EMG off-line by another method) because this is the measure of muscle activity that is relevant to task success. An index of covariation (IoC) (Eq. 1) was defined with values of $+1 /-1$ if the cursor position variability at time $t$ relative to trial onset is entirely constrained to the vertical/horizontal screen axes (positive/negative covariation in muscle space) and 0 if the variability is equal along these axes as follows:

$$
\operatorname{IoC}(t)=\frac{\sigma_{y}^{2}(t)-\sigma_{x}^{2}(t)}{\sigma_{y}^{2}(t)+\sigma_{x}^{2}(t)},
$$

where $\sigma_{x}^{2}(t)=\frac{1}{N} \sum_{i=1}^{N}\left[x_{i}(t)-\overline{x(t)}\right]^{2}$ and

$$
\sigma_{y}^{2}(t)=\frac{1}{N} \sum_{i=1}^{N}\left[y_{i}(t)-\overline{y(t)}\right]^{2}
$$

Here, $x(t)$ and $y(t)$ refer to the horizontal and vertical screen coordinates of the cursor at time $t$ relative to the start of trial $i$, and $\overline{x(t)}$ and $\overline{y(t)}$ are the mean of these instantaneous signals across trials. IoC analysis is closely related to the uncontrolled manifold analysis used by Kang et al. (2004), although in our formulation the sign is determined by the direction of muscle correlation regardless of the task-relevant dimension. To reveal the time course of correlation structure, IoC values were calculated individually for each time point during the hold period. Our subsequent analyses focused on a single time point in the trial (generally the beginning of the hold period). Separate IoC values were calculated for each target shape (vertical/horizontal ellipse and circle). We tested three different ways, combining data from the three target locations: the IoC for each target location were calculated separately and then the three values were averaged (1), or a single IoC value was calculated for the combined distribution of cursor positions defined relative to either the mean cursor position for each target (2) or the center of the target (3). We found these approaches to be comparable, so we present the results of method 3.

To quantify the evolution of task-specific muscle synergies we used multiple linear regression to relate IoCs (response variable) to two explanatory variables: block number $(1 \ldots 4)$ and target shape $(-1,0,1$, for horizontal, circular, and vertical targets, respectively), and their interaction according to the following:

IoC $=\beta_{o}+\beta_{1} \times$ block $+\beta_{2} \times$ target $+\beta_{3}$

$\times$ (block $\times$ target $),$

and computed $F$ statistics and the significance of each fit coefficient.

Intermuscular coherence. To assess the correlation between muscles within trials and determine its spectral components, we computed intermuscular coherence following the approach of Kilner et al. (2003). Coherence is a frequency-domain measure of the correlation between two signals that is bounded between 0 and 1. Raw EMG signals for each muscle were rectified and downsampled to $500 \mathrm{~Hz}$ to compute timefrequency spectrograms using 256 point overlapping windows. The time-frequency coherence spectrogram between rectified EMG signals was calculated according to the following:

$$
\operatorname{Coh}(t, f)=\frac{\left|\sum_{i=1}^{N} F_{1, i}(t, f) F_{2, i}^{*}(t, f)\right|^{2}}{\left|\sum_{i=1}^{N} F_{1, i}(t, f) F_{1, i}^{*}(t, f)\right|\left|\sum_{i=1}^{N} F_{2, i}(t, f) F_{2, i}^{*}(t, f)\right|},
$$

where $F_{j, i}(t, f)$ is the Fourier spectrogram of the rectified EMG signal from muscle $j$ at time $t$ relative to the start of trial $i$. In Equation $4, f$ denotes frequency and $N$ is the number of trials.

Significant intermuscular coherence implies that a fraction of the neural drive to those muscles arises from a common source (Rosenberg et al., 1989; Conway et al., 1995; Nielsen, 2002). In a nonparametric approach, all intermuscular coherence spectra corresponding to vertical and horizontal ellipse target conditions were thresholded at the $95 \%$ confidence level (Kilner et al., 2003) computed as follows:

$$
s=1-\alpha^{1 /(N-1)} .
$$

Coherence values larger than $s$ may be considered significantly different from zero with $p$ values smaller than $\alpha=0.05$. Thereafter, any timefrequency bin having values above that level were set to value 1 and those below or equal were given 0 and summed across subjects for each target shape. We used the McNemar's nonparametric test (McNemar, 1947) to identify time-frequency bins that showed a significant difference between the two orientations of target ellipse. Under the null hypothesis of no effect of target orientation, the number of subjects showing significant coherence in a given time-frequency bin only for one particular orientation ( $n_{V}$ and $n_{H}$ for vertical and horizontal ellipses) should be consistent with $N_{S}=n_{V}+n_{H}$ samples drawn from a binomial distribution with equal probabilities of 0.5 . Therefore, the two-tail significance level $p$ for rejecting the null hypothesis is given by the following:

$$
p=2 \times \sum_{i=0}^{k} \frac{N_{s} !}{i ! \times\left(N_{s}-i\right) !} \times 0.5^{i} \times 0.5^{N_{s}-i},
$$

where $k=\min \left(n_{V}, n_{H}\right)$ and $!$ denotes the factorial operator.

Feedforward model of corticospinal activity. We developed a feedforward model to explore how the motor system can exploit redundancy in the corticomuscular pathways to optimize performance in the presence of signal-dependent noise (SDN). In our model, seven neural units $U_{i=1 \ldots 7}$ (each representing a cortical population sharing the same output projections) drive two muscles $M_{i=1,2}$ with descending excitatory and/or inhibitory projections. For simplicity, we do not model specific 
A

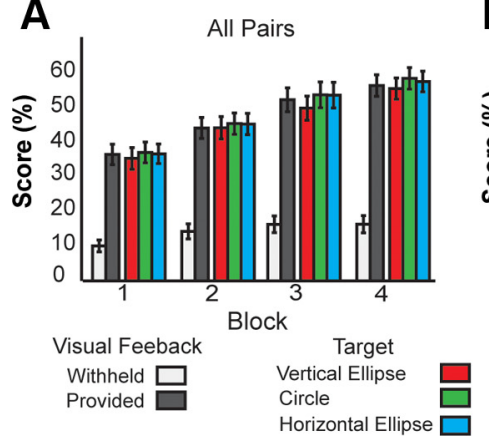

B

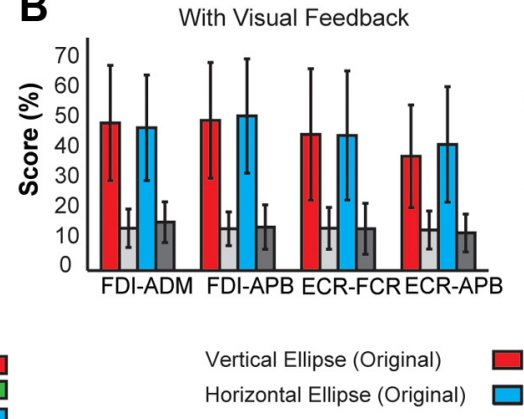

Without Visual Feedback

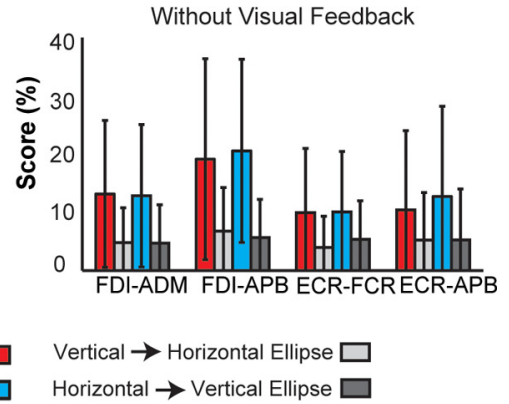

Figure 2. Analysis of performance score. A, Performance scores achieved by subjects in four training blocks averaged across all muscle pairs for different target shapes. Also shown are average scores for the three targets when visual feedback was present or absent (gray bars). $\boldsymbol{B}$, Actual scores for elliptical targets compared with the score that would have been obtained had the target orientation been reversed. Actual scores were consistently higher than for reversed orientations, even in the absence of visual feedback. Error bars represent SEM.

A

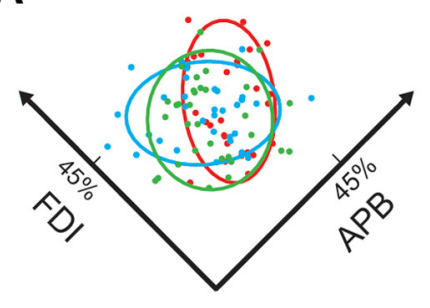

B

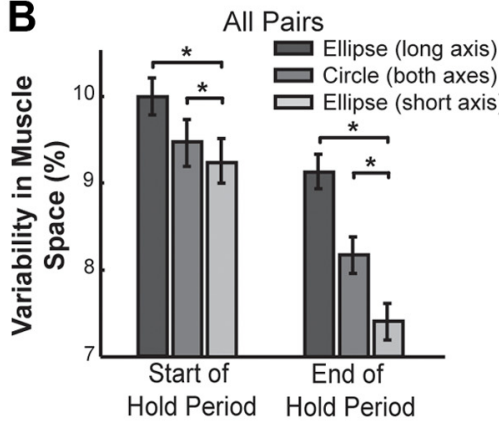

C

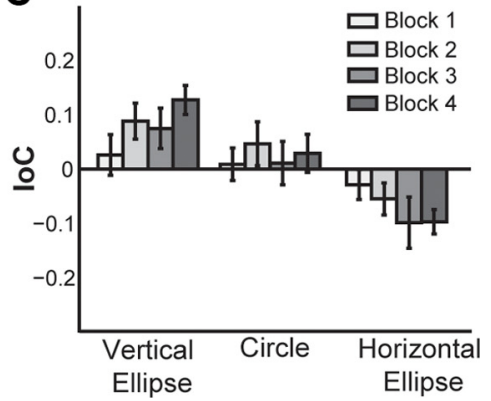

Figure 3. Analysis of trial-to-trial variability. $A$, Distribution of cursor position relative to target center at the end of the movement period in block 4 for a representative subject. The dots indicate single-trial data for horizontal (blue), vertical (red), and circle (green) target shapes. Corresponding fitted covariance ellipses are overlaid. $\boldsymbol{B}$, Average variability (SD) in the muscle space aligned to long and short axes of elliptical targets compared with the average of both axes for circular targets $\left({ }^{*} p<0.05\right)$. C, Emergence of target-modulated loC with training averaged over all CRM pairs at the start of the hold period. Error bars represent SEM.

properties of synaptic connections or mediating interneurons, and instead assume that muscle activity is determined by a weighted linear sum of neural activity. The weights are such that units $U_{i=1 \ldots 7}$ in isolation would move the cursor in directions $\theta_{i=1 \ldots 7}=i \pi / 8$. We do not include units with $\pi \leq \theta \leq 2 \pi$ in the model; these directions drive the cursor away from targets so activation of such units would always increase cursor variability and be suboptimal. For a given vector of neural activity distributed across $U_{i=1 \ldots}, 7$, the presence of neural and muscular SDN leads to a probability distribution of the resulting cursor position. Performance score was evaluated as the percentage of this distribution that overlapped with the target. We used the pattern direct search algorithm (Audet and Dennis, 2003) to find the neural activity vector that maximized the score for each target conditions. The fitting process for each pair of SDN values in $U$ or $M$ units was repeated for 10 independent runs of 100 repeated cursor movements for which the IoC values in each target condition were computed.

All data analysis was performed in MATLAB. Statistical tests were performed in SPSS 17.

\section{Results}

\section{Experiment 1}

Target-specific task performance improves with training

Performance scores improved significantly relative to block 1 $\left(r^{2}=0.67 ; p<10^{-5}\right.$; slope $=6 \%$ per block $)$ for all CRM pairs, as did the scores for interspersed trials without visual feedback $\left(r^{2}=\right.$ $0.16 ; p<10^{-3}$; slope $=2 \%$ per block) (Fig. $2 A$ ). Overall, in the absence of the visual feedback, scores were significantly lower (paired $t$ test; $t_{(15)}=-13.59 ; p<10^{-4} ; n=16$ ). The average performance scores achieved in elliptical and circular target trials were not different (two-way repeated-measures ANOVA; $\left.F_{(1,15)}=0.72 ; p=0.40 ; n=16\right)$. In principle, subjects could succeed at the task by aiming for the center of the target, regardless of its shape. To test whether target shape influenced subjects' strategy, we calculated off-line the score that subjects would have received had the orientation of target ellipses been reversed and compared this with the actual score obtained. Actual scores were consistently higher than for reversed orientations regardless of the availability of the visual feedback (Fig. 2 B). For each CRM pair, a 2 $($ Feedback $) \times 2$ (Orientation) repeated-measures ANOVA was performed confirming for all pairs main effects of the presence of the visual feedback and the actual versus reversed orientation of the target (FDI-ADM: $F_{(1,15)}=85.51, p<10^{-4}$; FDI-APB: $F_{(1,15)}=71.93, p<10^{-4}$; ECR-FCR: $F_{(1,15)}=68.54, p<10^{-4}$; ECR-APB: $\left.F_{(1,15)}=58.52, p<10^{-4}\right)$.

\section{Movement variability is buffered into less task-relevant dimensions}

We hypothesized subjects could exploit the redundancy afforded by elliptical targets by buffering trial-to-trial movement variability into the less relevant dimension (the long axis) to improve accuracy in the more relevant dimension (the short axis), as has been proposed for natural movements (Todorov and Jordan, 2002; Diedrichsen, 2007). Therefore, we examined the distribution across trials of instantaneous cursor position at the end of the movement and hold periods. The covariance ellipses in Figure $3 \mathrm{~A}$ for a representative subject late in training (block 4) suggest that cursor position at the end of the movement does indeed vary more along the long axes of elliptical targets. Figure $3 B$ compares, for all subjects with the four CRM pairs, the variability along the short and long axes of elliptical targets with the average variability 


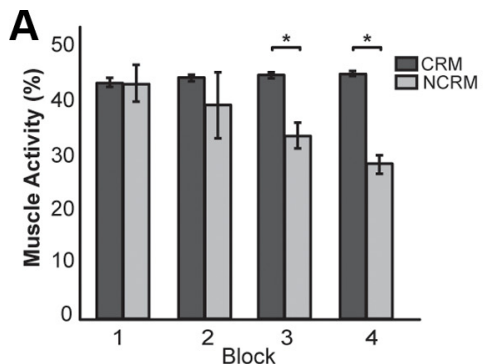

C

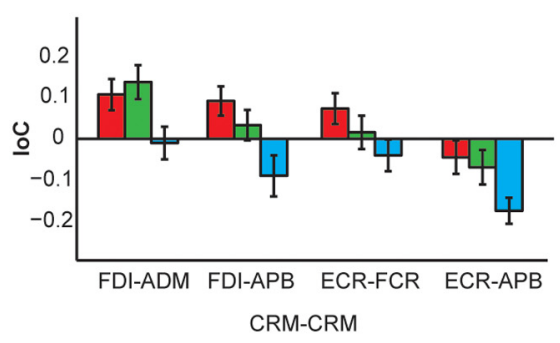

D

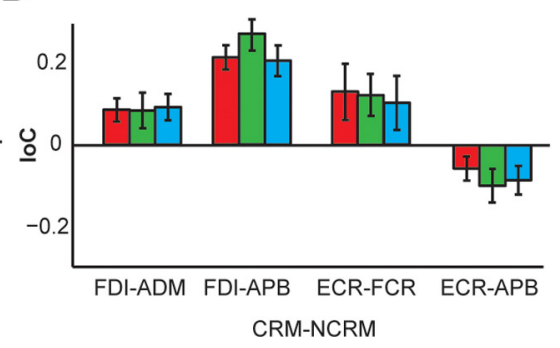

Figure 4. Comparison of the behavior of CRMs and NCRMs during myoelectric control. $A$, Average activity of CRMs and NCRMs across the four training blocks $\left({ }^{*} p<0.05\right)$. $\boldsymbol{B}$, Average lo $\mathrm{C}$ at the start of the hold period for all CRM-CRM, CRM-NCRM, and NCRM-NCRM pairs. C, Average loC for each CRM-CRM muscle pair across the three targets computed at the start of the hold period. $D$, Average loC for the same muscle pairs in cases in which only one of the pair was a CRM. Error bars represent SEM.

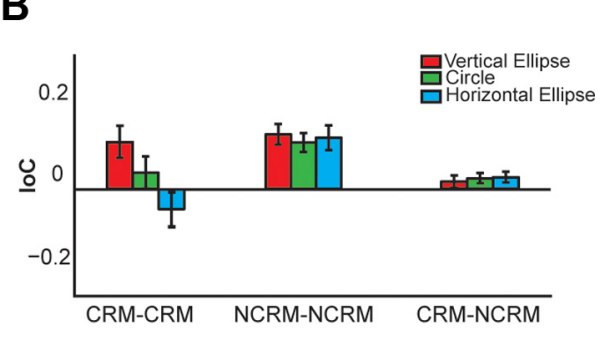

uncontrolled manifold) compared with task-relevant dimensions has previously been interpreted as indicating a synergy (Latash et al., 2002). Our results suggest that, with practice, subjects learn to coordinate appropriate synergies comprising arbitrary muscle pairs according to abstract task demands that vary on a trial-totrial basis.

\section{Activity of non-cursor-related muscles}

Target modulation of IoCs suggests that arbitrary CRM pairs can be combined into appropriate synergies according to the orientation of target ellipses. This is evidence for a "high-level" interpretation of synergies arising from constraints imposed by specific task features and suggests that a large number of synergies can be constructed from a small number of muscles. However, it remains possible that the emergence of target-dependent correlation structure between pairs of CRMs in fact reflects the recruitment of synergies that span multiple muscles in addition to those controlling the task.

along both axes of the circular target. Overall, the variability of cursor position was comparable for both elliptical and circular targets (paired $t$ test, start of hold: $t_{(15)}=1.18, p=0.25, n=16$; end of hold: $t_{(15)}=1.28, p=0.21, n=16$ ), which were of equal area. However, variability along the more relevant short axis was not only smaller than along the less relevant long axis (paired $t$ test, start of hold: $t_{(15)}=-7.13, p<10^{-4}, n=16$; end of hold: $\left.t_{(15)}=-12.47, p<10^{-4}, n=16\right)$, but also lower than for the circular targets (paired $t$ test, start of hold: $t_{(15)}=-2.04, p=0.05$, $n=16$; end of hold: $t_{(15)}=-9.29, p<10^{-4}, n=16$ ). In other words, by relaxing the constraint on accuracy aligned with the less relevant dimension of the task, subjects made smaller errors along the more relevant dimension when compared with the circular target condition (which constrained both dimensions). However, perhaps remarkably, overall task performance closely matched that for the similar area circular targets, as noted above. This behavior is consistent with computational theories proposing that synergistic muscle activity allows the motor system to exploit redundancy to improve task-relevant accuracy (Latash et al., 2001; Todorov and Jordan, 2002; Diedrichsen, 2007).

IoC analysis reveals emergence of abstract, task-specific synergies To quantify synergistic muscle activation, we defined an IoC (Eq. 2) with values of $+1 /-1$ if endpoint variability is entirely constrained to the vertical/horizontal screen axes (positive/negative covariation in muscle space) and 0 if variability is equal along these axes. Figure $3 C$ shows the emergence of positive and negative IoC values appropriate for the elliptical targets for pooled data from all CRM pairs across the four training blocks, paralleling the improvement in performance score (Fig. $2 A$ ). To test the significance of the effect of training on task-specific IoC, we used a multiple linear regression model (Eq. 2) with parameters reflecting the effect of target shape $\left(\beta_{2}\right)$ and its interaction with block number $\left(\beta_{3}\right)$. We found a significant main effect of target shape $\left(\beta_{2}=0.077 ; t=10.17 ; p<10^{-4}\right)$. Moreover the interaction between target shape and block number (indicative of a learning effect) was also significant $\left(\beta_{3}=0.022 ; t=3.32 ; p=\right.$ 0.01 ). Higher variability in task-irrelevant dimensions (the
Since the task does not impose high-level constraints on these NCRMs, their correlation with CRMs could reveal the existence of such low-level, hardwired synergies. We therefore examined patterns of activity in NCRMs during myoelectric control. The low-level synergy explanation makes three predictions. First, if the acquisition of accurate myoelectric control involves learning to recruit multimuscle synergies that involve both CRMs and NCRMs, we would expect to see activity in NCRMs persist or increase with learning. Second, we would expect to see correlation structure between CRMs and NCRMs. Third, if the target-dependent modulation of CRM correlation structure arises from task-dependent modulation of these multimuscle synergies, we would expect the covariation of CRMs and NCRMs also to be target modulated.

Figure $4 \mathrm{~A}$ shows that the activity of NCRMs during the hold period decreases progressively with training over the same period that target-dependent correlation structure between CRMs appears (paired $t$ test, $n=16$; block 3: $t_{(15)}=-3.37, p=0.004$; block 4: $\left.t_{(15)}=-6.15, p<10^{-5}\right)$. This seems incompatible with the use of multimuscle synergies to complete the task, instead suggesting that, while maximizing performance score, subjects also try to minimize unnecessary muscular effort (Diedrichsen et al., 2010). To examine correlation structure, we computed the IoC at the start of the hold period for each target orientation presented in the central position. Average IoC values for every possible combination of CRM-CRM (4 pairs), NCRM-NCRM (12 pairs), and CRM-NCRM (24 pairs) and are shown in Figure 4B. NCRM-NCRM pairs exhibited positive IoC scores because the declining activity of these unconstrained muscles with training produced a positive trial-by-trial correlation. This effect disappeared for CRM-NCRM pairs since the activity of the CRM was maintained through training. The remaining trial-by-trial variability between these pairs showed a small positive covariation, which reached significance for circle $(p=0.04)$ and horizontal ellipse $(p=0.03)$ targets (one-sample $t$ test; $n=16)$. In addition, we performed a one-way ANOVA test (repeated measures; $n=16$ ) for each of the CRM-CRM, NCRM-NCRM, and 
CRM-NCRM cases separately to test the target dependency of the IoC values. Only in the case of CRM-CRM were the IoCs target dependent: CRM-CRM $\left(F_{(2,30)}=5.74 ; p=0.008\right)$, NCRM$\operatorname{NCRM}\left(F_{(2,30)}=0.36 ; p=0.71\right)$, and CRM-NCRM $\left(F_{(2,30)}=\right.$ $0.917 ; p=0.41)$.

To explore further the interaction between high- and lowlevel constraints on muscle synergies, we directly compared IoC values for CRM-CRM pairs controlling the task (Fig. 4C) with IoCs for the same pairs when one muscle was a NCRM and therefore unconstrained by the task (Fig. 4D). Interestingly, certain pairs showed high correlation when only one muscle was constrained. For example, the IoC for the FDI-APB pair (calculated during the FDI-ADM and ECR-APB control conditions) was on average $\sim 0.2$ for all target conditions. This may result from the use of a "low-level" synergy involving both muscles, or simply reflect some biomechanical coupling of muscles to help stabilize the hand posture. In either case, these constraints are not absolute since when both muscles controlled the task (Fig. 4C) a targetdependent pattern was observed between FDI-APB, with a negative IoC for the horizontal ellipse target appropriate for high-level constraints. In fact, for all CRM pairs, IoC was greater for vertical than horizontal ellipse targets, and in only one of eight combinations was the sign inappropriate for the orientation of elliptical target (ECR-APB, vertical ellipse). When analyzed individually with the regression model (Eq. 2), the effect of target shape $\left(\beta_{2}\right)$ was in all cases significant while the learning term $\left(\beta_{3}\right)$ was significant for the pairs FDI-APB $\left(\beta_{3}=0.04 ; t=2.721 ; p=\right.$ $0.026)$ and ECR-FCR $\left(\beta_{3}=0.048 ; t=2.845 ; p=0.021\right)$. By contrast, when only one muscle was a CRM (Fig. $4 D$ ), none of the pairs showed a significant effect of target shape $(p>0.05)$. Together, these results demonstrate that, even if low-level synergies may be used when the task allows, these do not represent hard constraints and can be readily overridden as required by highlevel task demands.

\section{Influence of visual feedback on muscle synergies}

Figure $5 A$ compares average IoC values for cursor distributions through the hold period when the visual feedback was available. The influence of target shape on IoC increased during the hold period for both elliptical target orientations. IoCs improved from the first to the last time instants during the hold period for both the vertical $\left(0.07-0.17\right.$; paired $t$ test; $t_{(15)}=4.29 ; p=10^{-3} ; n=$ $16)$ and the horizontal ellipse target conditions $(-0.07$ to -0.23 ; paired $t$ test; $\left.t_{(15)}=6.202 ; p=10^{-3} ; n=16\right)$. This suggests that visual feedback of errors during the hold period contributes to shaping the correlation structure (see Experiment 2). However, even in the absence of the visual feedback, IoC values at the end of the hold period showed a significant (one-way repeatedmeasures ANOVA; $F_{(2,30)}=0.384 ; p=4.25 \times 10^{-5} ; n=16$ ) effect of target orientation (Fig. $5 B$ ), suggesting that visual feedback alone cannot account for target-dependent modulation of IoC. Interestingly, although the IoC was still higher in the case of vertical target ellipses, in the absence of visual feedback IoC values were biased in the direction of positive covariation, such that the minimum IoC observed (in the case of horizontal ellipse targets) was approximately zero.

\section{A feedforward model of structured variability}

In the absence of feedback, correlation structure in movement variability could arise from central sources of noise propagating through divergent projections to muscles. For example, trial-totrial variation in the activity of a neuron that drives both CRMs would lead to positive covariation in muscle space, which may be
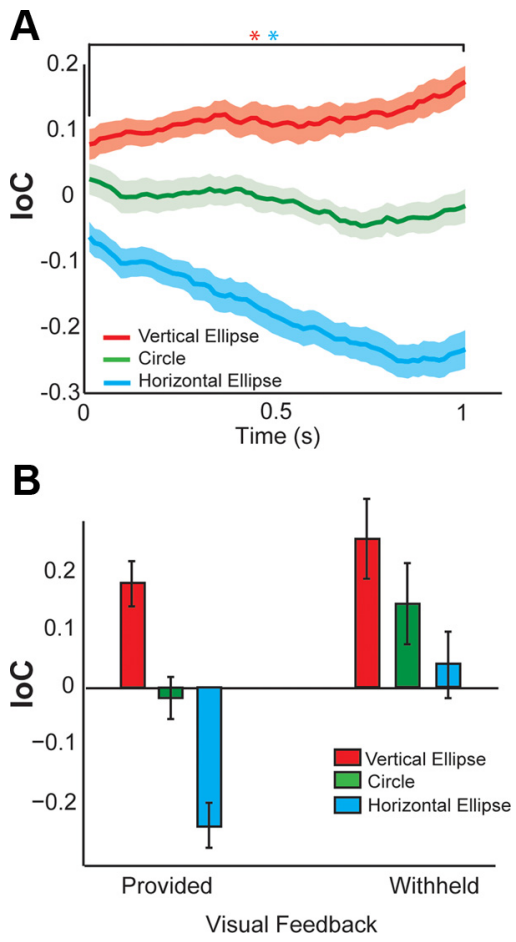

Figure 5. Influence of visual feedback on loCs. $\boldsymbol{A}$, Development of target-modulated lo through the hold period in the presence of visual feedback $\left({ }^{*} p<0.05\right)$. $\boldsymbol{B}$, Comparison of target-modulated $\mathrm{l} C \mathrm{C}$ at the end of the hold period in the presence and absence of visual feedback. Error bars represent SEM.

appropriate for the vertical ellipse target. We developed a simple feedforward model (Fig. 6A) to elaborate this idea. We assumed that the activity of populations of neurons with distinct patterns of projections to muscles is subject to SDN (Harris and Wolpert, 1998). Any given pattern of activity across these populations therefore results in a probability distribution in muscle space, reflecting the likelihood of generating that muscle activity in the presence of noise. The overlap of this distribution with a particular target area estimates the proportion of time that the cursor will be within target, equivalent to the score subjects received in our task. Therefore, we optimized the feedforward drive to muscles in our model by maximizing this overlap using numerical methods (see Materials and Methods). Because we did not find strong evidence for the recruitment of multimuscle synergies including NCRMs, we only modeled projections to CRMs. Note, however, that if some of the projection neurons also have outputs to NCRMs, this could explain positive correlations between CRM-NCRM pairs.

The feedforward model verified our basic premise that different target shapes would lead to different optimal solutions (Fig. $6 B)$. A consequence of SDN is that accuracy is improved when effort is distributed across the different neuronal populations. For a circular target, the predicted distribution obeys truncated cosine tuning in muscle space; for this special case, the same solution can also be obtained analytically (Todorov, 2002). In the case of the vertical ellipse target, the tuning function was narrower, with elevated activity of units $U_{i=3,4,5}$ (shaded in Fig. $6 A, B)$, which facilitate both muscles. For horizontal targets, the model predictions were reversed, with lower common drive and higher activity in units $U_{i=1,2,6,7}$, which facilitate one muscle only. Interestingly, when IoC values were calculated for the resultant probability distributions in muscle space, the target dependence was similar to our experimental results in the absence of visual 
A
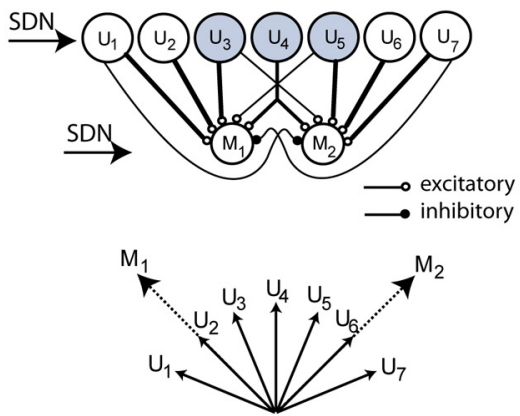

C

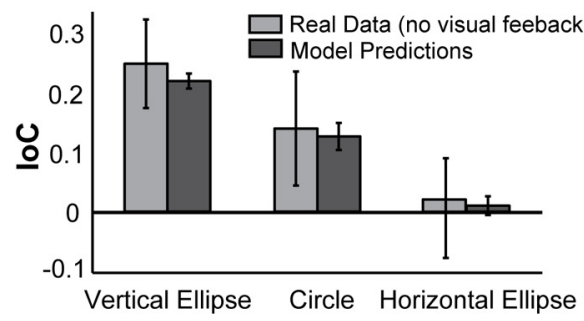

B
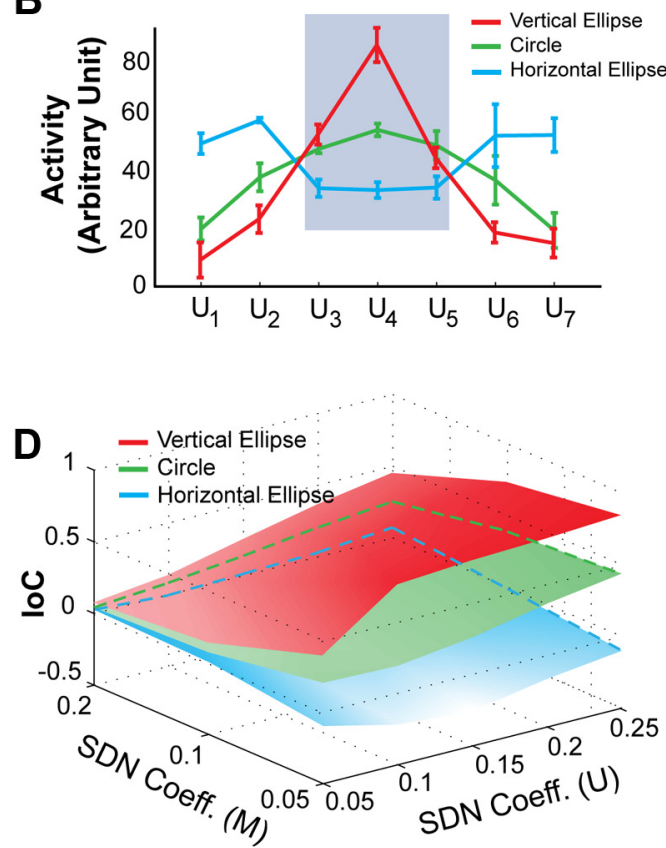

Figure 6. A feedforward model of task-dependent correlation structure. $A$, The open and filled links from the input units $U_{i=1 \ldots 7}$ to muscles $\left(M_{1}, M_{2}\right)$ depict excitatory and inhibitory connections, respectively. The line thickness is proportional to the connection weight. The action of these connections in the cursor space is shown underneath. $\boldsymbol{B}$, The computed activity of each of the input units to maximize the performance score. SDN is buffered into dimensions of positive muscle covariance by common inputs (shaded). $\boldsymbol{C}$, Computed loCs from measured (without visual feedback) and simulated data. $D$, The target dependence and positive bias of lo $C$ values was unaffected by the precise choice of SDN coefficients of the neural units ( $U$ ) or muscles ( $M$ ).

feedback (Fig. 6C); while IoC was highest in the case of the vertical ellipse, it was also positive for the circular target and around zero for the horizontal ellipse. This asymmetry arises because although the horizontal ellipse penalizes positively correlated variability, both muscles must nevertheless be coactivated to reach the central target. In this simulation, we chose those neural and muscular noise coefficients $(0.15$ and 0.2 , respectively, for neural and muscular units) to give IoC values and average scores comparable with our recorded data. However, Figure $6 D$ shows the main results of the model (target-dependent IoC values biased in the positive direction) were unaffected by the precise choice of noise coefficients. These simulations were performed for the central target only, but comparable results were obtained for side targets also.

Common cortical drive is modulated by target shape

The model described above predicts that feedforward divergent drive to CRMs should be modulated by target shape, despite the fact that the average muscle activity is in each case the same. We speculated that it might be possible to find evidence for such an effect by analyzing the correlation structure between EMG signals at finer temporal resolutions than were present in the smoothed cursor position. Figure $7 A$ shows time-domain crosscorrelations between rectified EMG during the hold period for an example subject. The broad central peak is indicative of physiological synchrony between motor units arising from common drive, while side bands suggest the presence of oscillatory synchrony. These cross-correlation features were consistent for each target shape across all subjects. However, it is difficult to assess target-dependent variation of common drive at specific frequencies using time-domain analyses. Therefore we calculated intermuscular coherence in the frequency domain from the rectified EMG, since this can reveal common oscillatory drive to muscles. In our task, we found significant intermuscular coherence between the muscle pair FDI and APB, so we examined whether this was target dependent. Figure $7 B$ shows the spectrogram of timefrequency bins in which coherence differed significantly between vertical and horizontal target ellipses. We found a significant elevation of coherence for the vertical target orientation during the hold period. Figure $7 C$ shows that this effect of target shape was significant only in the beta band around $20 \mathrm{~Hz}$, and not in the alpha band around $10 \mathrm{~Hz}$ (ANOVA beta band: $F_{(2,30)}=7.18, p=$ 0.003; alpha band: $\left.F_{(2,30)}=0.098, p=0.907 ; n=16\right)$. Post hoc Bonferroni's correction revealed the difference in the percentage of significant time-frequency bins between the vertical and horizontal ellipse target conditions was statistically significant (twotailed $t$ test; $\left.t_{(15)}=4.10 ; p=0.001 ; n=16\right)$. Figure $7 D$ shows that the target modulation of beta-band coherence was only present during the hold period of the task. Furthermore, beta-band taskmodulated intermuscular coherence of FDI-APB muscles was only observed when they were used as a CRM pair. When they formed a NCRM-CRM or NCRM-NCRM pair, no task-related modulation in either alpha or beta bands was detected.

Coherence in the beta-frequency range observed during tonic contractions of distal musculature is believed be of cortical origin (Farmer et al., 1993). Corticospinal neurons are incorporated into the networks that generate beta oscillations (Jackson et al., 2002) and corticomuscular coherence at this frequency depends on the integrity of the corticospinal tract (Farmer et al., 1993; Hansen et al., 2005; Norton and Gorassini, 2006). Intermuscular coherence may reflect synchrony between corticomotoneuronal cells with common muscle fields (Jackson et al., 2003), divergence in the descending projections of individual corticomotoneuronal cells (Shinoda et al., 1981; Lawrence et al., 1985; Buys et al., 1986), cortical inputs to spinal premotor interneurons contacting multiple motoneuron pools (Takei and Seki, 2010), or some combination of all these. In any case, our results suggest a cortical origin for the task-dependent common drive to muscles

(Fig. 5A, $U_{i=3,4,5}$ ). 
This simple feedforward model of divergent corticospinal drive, while accounting for behavior in the absence of visual feedback, failed to describe several features of the data when visual feedback was present. In particular, the observed negative correlation between muscles was not predicted by feedforward mechanisms alone. Therefore, in Experiment 2, we examined whether visuomotor feedback mechanisms also contributed to the emergence of task-dependent correlation structure in muscle activity.

\section{Experiment 2}

\section{Task-dependent visuomotor feedback} control

In Experiment 2, we perturbed the position of the cursor to examine feedback corrections made by subjects (Fig. $8 \mathrm{~A}$ ). If subjects are attempting to maintain the cursor at a defined location in the twodimensional muscle space (i.e., the center of the target), then the correction should occur in the direction opposite to the applied perturbation. Therefore, a perturbation aligned to the axis of one of the controlling muscles should elicit a response in that muscle alone. However, if visuomotor feedback acts predominantly along the dimension in the muscle space that is most relevant to task success, then a response is predicted in the other muscle also (Fig. $8 B$ ). Such a minimum intervention strategy may be optimal if there is a cost associated with large corrective movements and leads to an accumulation of errors in task-irrelevant dimensions (Todorov and Jordan, 2002). Since the task-relevant dimension is determined by high-level constraints (in this case, target shape), a strong prediction is that the response in the orthogonal muscle should be modified under different task constraints (vertical and horizontal ellipses). These predictions are depicted in Figure $8, C$ and $D$.

EMG activity was analyzed according to two factors: target shape (vertical vs horizontal ellipse) and perturbation (parallel or orthogonal to the muscle axis). When the perturbation was parallel to the muscle axis, activity was reduced by $\sim 5 \%$ regardless of the target shape (Fig. 8E,F). However, the key test of our two feedback hypotheses was when the perturbation was orthogonal to the muscle axis. In this case, a target-dependent response was observed, with a decrease of $\sim 2 \%$ for horizontal ellipse targets and an increase of $\sim 2 \%$ for vertical ellipse targets (Fig. $8 E, F$ ). This is consistent with a minimal feedback controller acting preferentially along the more task-relevant axis in the muscle space. Figure $9 A$ details the changes in the activity of the muscles (parallel or orthogonal to the perturbation) with respect to their corresponding baseline activity (averaged over $100 \mathrm{~ms}$ preceding the perturbation) for individual subjects. For the muscle acting parallel to the perturbation (filled circles), the data cluster around the line of equal response for both target orientations. However, for the muscle acting orthogonal to the perturbation, data for 17 of 20 subjects fall above the line, indicating a greater response for the vertical target. Note, however, that the magnitude of the response in the orthogonal direction was in general less than in the parallel direction. Thus, feedback control is not entirely constrained to a single dimension in the task space. This likely reflects
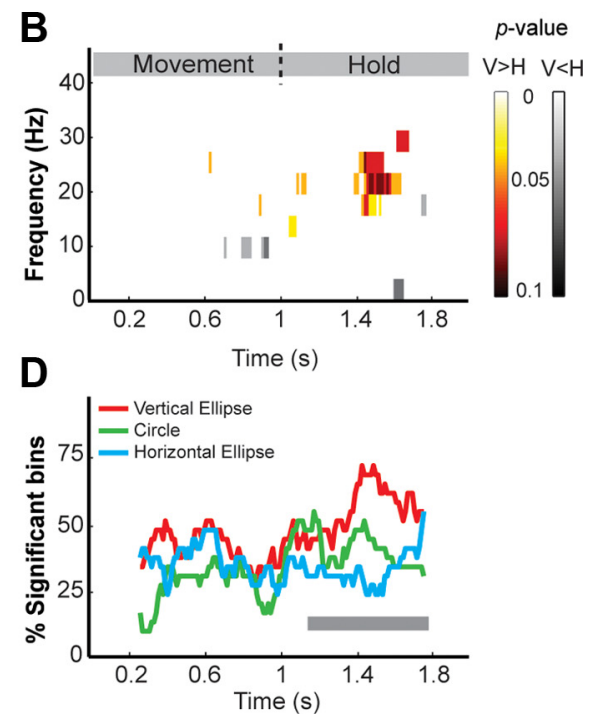

Figure 7. Task-related modulation of beta-band intermuscular coherence. $\boldsymbol{A}$, Time-domain cross-correlation between FDI and APB hold period activity from a typical subject. The asterisks indicate statistically significant peaks/troughs ( $>3$ SDs from baseline). $\boldsymbol{B}$, Time-frequency plot of statistical significance of intermuscular coherence modulation by target shape for FDI-APB muscle Beta band intermuscular coherence is significantly modulated by target shape in contrast to alpha band. $\boldsymbol{D}$, Time course of significant beta-band $(19-25 \mathrm{~Hz}$ ) intermuscular coherence for different target shapes.

the fact that our elliptical targets, despite being wider in one dimension, nevertheless penalize large deviations along this axis. Hence, this dimension is not entirely irrelevant to task success.

The corrective response to the visual perturbation emerged on average $150 \mathrm{~ms}$ after the onset perturbation onset in both perturbed and unperturbed muscles and for both target orientations. This latency is comparable with involuntary visual reflexes that have been described during natural reaching (Franklin and Wolpert, 2008).

\section{Amplitude of task-dependent visuomotor responses increases as} performance improves

To examine the effect of training, we divided perturbation trials into those that occurred in the first and second halves of the experimental session. We found that the magnitude of response in the muscle acting orthogonal to the perturbation direction was consistently larger in the second half of the session (Fig. 9B). A $2 \times 2$ ANOVA revealed significant effects of target shape $\left(F_{(1,19)}=53.25 ; p<10^{-4}\right)$ and its interaction with training pe$\operatorname{riod}\left(F_{(1,19)}=7.18 ; p<0.01\right)$ on the orthogonal muscle response. Over the same period, subjects' performance in unperturbed trials improved from 32 to $41 \%$ (paired $t$ test; $t_{(1,19)}=5.22 ; p<$ $10^{-4} ; n=20$ ).

\section{Experiment 3: reflex responses to peripheral stimuli are not task dependent}

In Experiment 3, we extended the logic of the previous experiment to look for target-dependent feedback responses to somatosensory stimulation in the absence of visual feedback. Task-dependent long-latency reflexes have been described in other tasks (Dietz et al., 1994; Nadler et al., 2000). We hypothesized that proprioceptive signals following peripheral stimulation would be interpreted as errors, and that subjects might learn to correct these errors via modulation of reflex pathways (Matthews, 1991). Therefore, we looked for target-dependent reflex responses in ADM (innervated by the ulnar nerve) after electrical stimulation of the median nerve, and target-dependent responses 
A

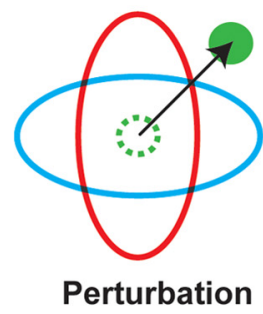

B

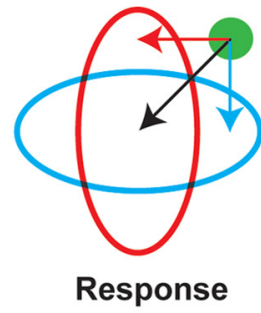

C

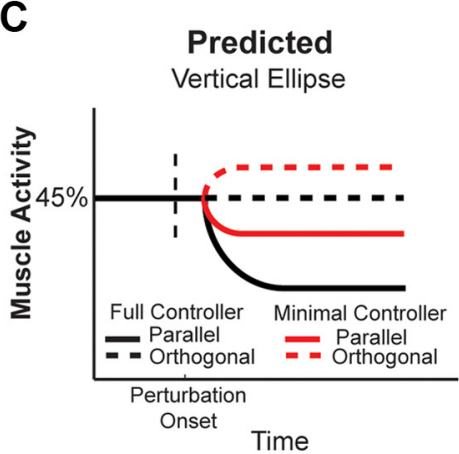

E

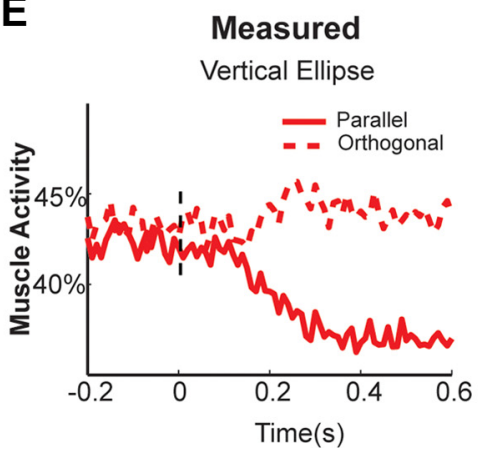

D

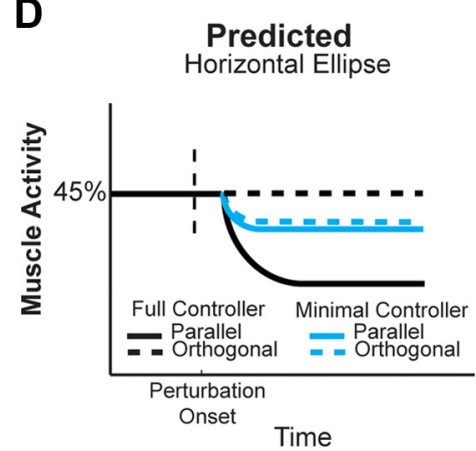

$\mathbf{F}$

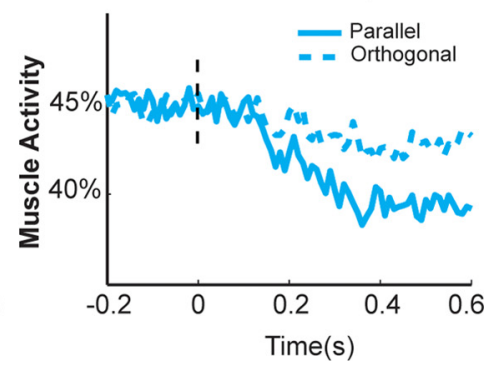

Figure 8. Response to visual perturbations. A, Visual displacement of the cursor for vertical and horizontal ellipse target shapes. $\boldsymbol{B}$, A full two-dimensional muscle space controller corrects the cursor position in the direction opposite to the applied perturbation (black arrow) but a minimal controller compensates mainly along the task-relevant dimension in the muscle space (red and blue arrows for vertical and horizontal ellipse targets, respectively). Predicted $(\boldsymbol{C}, \boldsymbol{D})$ and measured $(\boldsymbol{E}, \boldsymbol{F})$ muscle responses to visual displacement of the cursor. The direction of response in the muscle acting orthogonal to the perturbation (dashed trace) depends on the orientation of the target in accordance with the minimum intervention principal.

in APB (innervated by the median nerve) following stimulation of the cutaneous nerve of the little finger. Raw EMG signals were rectified, translated into screen coordinates (Eq. 1), and downsampled to $500 \mathrm{~Hz}$ before averaging across trials. We saw a number of features in the EMG signal, reflecting spinal and longlatency responses (Fig. 10). However, in no case was the response modulated by target condition. Paired $t$ test analysis in time windows (1) to (7) did not reveal any statistically significant difference in the EMG responses to electrical stimulations (paired $t$ tests; $p>0.05 ; n=11$ ). Therefore, unlike the response to visual perturbation, we did not find evidence of target-dependent reflex responses to peripheral stimulation in this myoelectric control task.

\section{Discussion}

Muscle synergies as optimal solutions for high-level constraints

Our primary finding is the emergence with training of structured variability between arbitrary pairs of upper-limb muscles used to control a myoelectric interface. Moreover, the magnitude and sign of covariation was modulated appropriately for target di- mensions that varied on a trial-by-trial basis. This was true for CRM pairs that were natural synergists (e.g., FDI-APB), antagonist muscles (ECR-FCR), and pairs with no obvious relationship (ECR-APB). Structured variability has previously been interpreted as a signature of synergies (Latash et al., 2002). If so, then our results demonstrate that, rather than being confined to a small number of basic primitives, the human hand can generate a wide range of synergies appropriate for many learned, skilled behaviors. This is inconsistent with the suggestion that low-level synergies (or M-modes) simplify the control of a redundant effector since the dimensionality of this "synergy space" is larger than the number of individual muscles; if anything, the degrees of freedom problem is exacerbated! Instead, our results are consistent with synergies emerging from optimization for high-level task requirements, in this case an accuracy constraint imposed by target shape. Since variability in the more task-relevant (short) axis for elliptical targets was reduced relative to circular targets, we conclude that subjects improved task performance by exploiting leniency in the less task-relevant (long) axis; in other words, synergies "buffered" noise into the uncontrolled manifold. While correlation structure in movement variability has been observed previously with finger force production (Kutch et al., 2008; O'Sullivan et al., 2009; Park et al., 2010), unimanual pointing (Knill et al., 2011), bimanual pointing (Domkin et al., 2002), and grasp (Todorov and Ghahramani, 2004), these studies do not preclude hardwired controllers specialized for naturalistic movements. By contrast, we demonstrated that new synergies emerge within one session of training on an abstract task using arbitrary pairs of muscles as the optimal way to exploit redundancy and maximize accuracy in the presence of neuromotor noise.

\section{Feedforward generation of synergies}

Correlation structure characteristic of synergies can arise from divergent descending pathways since variability in the motor command will result in correlated activity in the muscles. By varying the relative contribution of such divergent drive, structured variability could be shaped through a feedforward control strategy. Evidence for this mechanism was found in the modulation of intermuscular coherence, at frequencies that were too high to appear in the smoothed cursor position and thus could not be influenced by visual feedback. Target-modulated coherence was restricted to the beta band, suggesting subjects exploited divergence in the descending corticospinal pathway as a feedforward strategy to minimize the influence of central sources of neuromotor noise on task-relevant dimensions.

Divergence in the corticospinal system is often assumed to coordinate muscles during movement (Buys et al., 1986), but the 
computational benefits of this organization are not always apparent. Divergent corticospinal projections may be comparable with hardwired motor primitives wherein the muscle field of each cell represents a different synergy. Drew et al. (2008) argue that, during locomotion in the cat, descending corticospinal activity associated with obstacle avoidance acts via the same discrete synergies that are hardwired into spinal central pattern generators. However, the neural activity that enacts even simple hand movements in primates is distributed across cortical populations with monosynaptic connections to a large variety of diverse muscle fields (Fetz and Cheney, 1978; Buys et al., 1986; Jackson et al., 2003). It is not clear that coordinating all these "synergies" should be easier than coordinating the individual muscles. We believe that rather than a hardwired solution to the degrees of freedom problem, the extensive convergence and divergence in the corticospinal system is best understood as providing a rich substrate for optimizing movements in the presence of central neuromotor noise. Put simply, the computational costs associated with a highdimensional control space are offset by the flexibility afforded by this additional level of redundancy. Modulating the contribution of divergent populations to the overall motor output allows noise to be buffered into different uncontrolled manifolds within the effector space, as appropriate for a variety of dynamic high-level task constraints.

The model in Figure 6 illustrates these ideas in the context of our task. While all the tuning curves (Fig. $6 B$ ) take the cursor to the same average location in muscle space, each is associated with a variability structure that is suited to different highlevel goals (target shapes). It remains to be seen whether more sophisticated models of divergent projections to upper-limb muscles could predict the distributed patterns of cortical activity seen during naturalistic hand movements. However, this simple model does make predictions that could be tested experimentally, for example, that cosine tuning should breakdown when reaching to noncircular targets (Fig. $6 B$ ). The model also predicts that for circular targets, variability between CRMs should be positively correlated. This was true for our task only in the absence of visual feedback, indicating a second mechanism modulates correlation structure based on visual feedback.

\section{Feedback generation of synergies}

Effector space variability can be shaped by task-specific feedback if error correction occurs predominantly along task-relevant di-
A

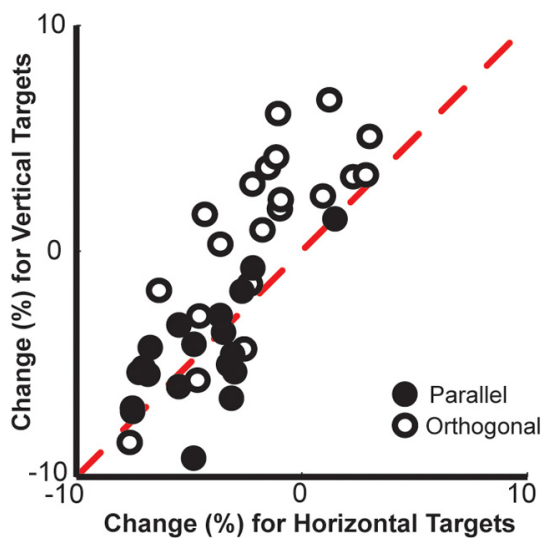

B

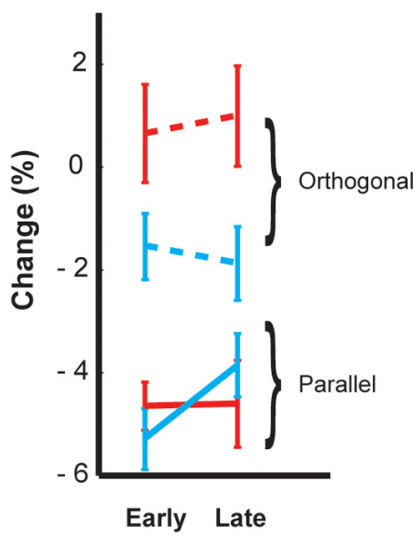

Figure 9. Summary of visual perturbation data. $A$, Percentage change in the activity of muscles acting parallel and orthogonal to the direction of the perturbation for individual subjects. $\boldsymbol{B}$, Target-modulated response in the orthogonal muscle increases with training. All changes are expressed as a percentage of baseline activity preceding the perturbation. Error bars represent SEM.
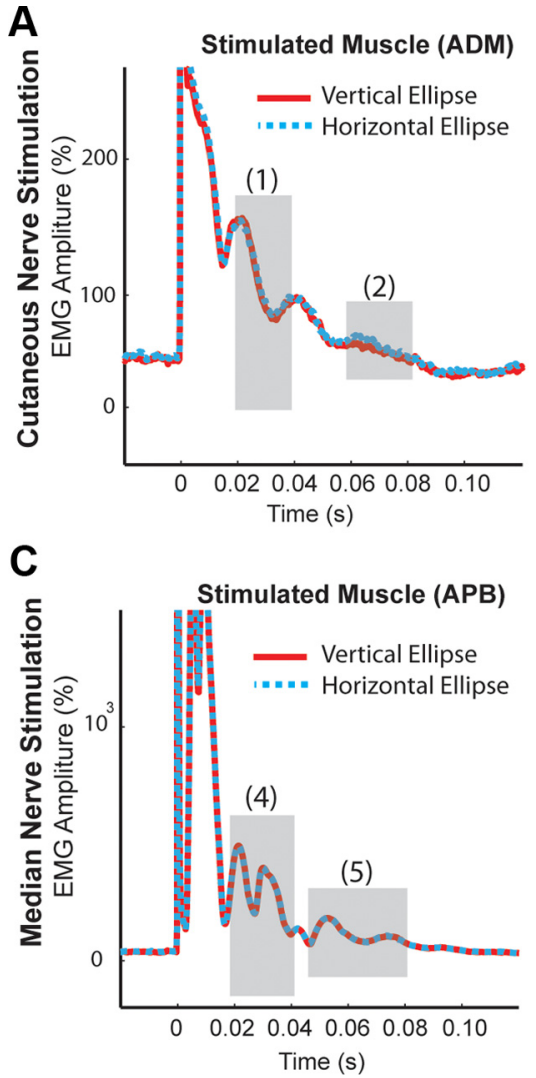

B

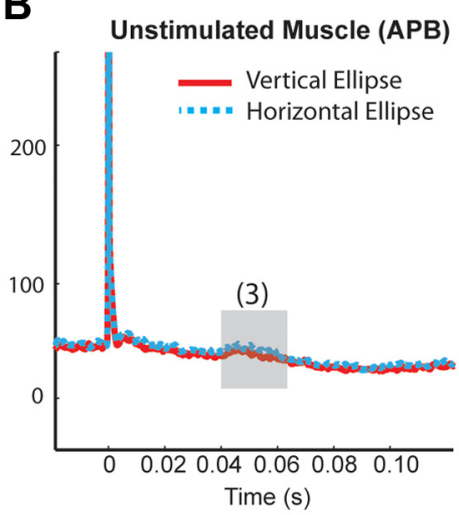

D

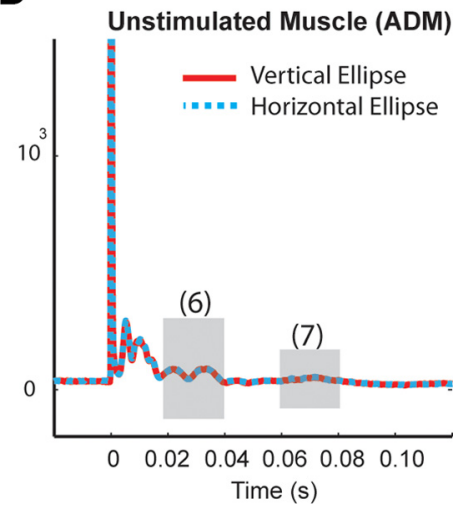

Figure 10. EMG responses to cutaneous $(\boldsymbol{A}, \boldsymbol{B})$ and median $(\boldsymbol{C}, \boldsymbol{D})$ nerves stimulations. Despite observation of several spinal and long-latency responses (1) to (7), in none of the shaded intervals was there a significant difference between the vertical and horizontal target conditions $(p>0.05)$.

mensions (Todorov and Jordan, 2002). We found evidence for minimum intervention control after visual perturbation along the axis of one CRM. If the motor system controlled each muscle independently, then a corrective response would be expected only in the CRM that acts parallel to the perturbation to bring the cursor back to the center of the target. Instead, we saw a targetdependent response in the orthogonally acting muscle, consistent with the minimal intervention required to return the cursor into the target. The latencies of responses in perturbed and unperturbed 
muscles were comparable, and similar to task-dependent involuntary visuomotor responses described by Franklin and Wolpert (2008).

Interestingly, while standard formulations of optimal feedback control minimize muscular effort to maximize accuracy (Diedrichsen et al., 2010), this is not quite consistent with our results. For instance, in the case of the vertical target, the perturbation caused an increase in muscle activity. Rather, our results suggest minimization of the correction to the motor command, which is presumably represented at a higher level in the motor system (Archambault et al., 2009), and may itself be subject to a form of signal-dependent noise.

\section{Role of peripheral feedback}

Experiment 3 examined the role of afferent feedback for minimum intervention control in the absence of visual feedback. Task-dependent reflexes that are distributed across muscles, for example mediated by reciprocal inhibitory interneurons, could modulate structured variability according to target shape. In natural movements, transcortical reflex components can be modified by high-level task-demands (Rothwell et al., 1980; Day and Lyon, 2000; Nadler et al., 2000; Kurtzer et al., 2008). However, although we resolved a number of reflex components in both CRMs, none revealed any target dependence. While it is possible that small differences may have remained unresolved, our negative result is consistent with our previous finding that proprioceptive information may be relatively unimportant for learning to control myoelectric interfaces (Radhakrishnan et al., 2008; Jackson and Fetz, 2011).

\section{Constraints on the flexibility of muscle synergies}

We found that the cortical control of hand muscles was sufficiently flexible to form appropriate task-specific synergies, but there are several important caveats to make. First, we do not rule out the existence of hardwired primitives for hand muscles encoded in spinal circuits but suggest that if they do exist they can be rapidly overridden by corticospinal projections. Furthermore, despite the diversity of corticomotoneuronal projections, not all muscle field combinations are equally likely (Buys et al., 1986), with overrepresented patterns presumably reflecting useful synergies for common behaviors like grasping. These neuroanatomical constraints may limit the optimality of myoelectric control under some circumstances. For example, we only studied relatively weak muscle contractions but there is evidence that during maximal contractions of individual fingers neighboring digits can become enslaved (Zatsiorsky et al., 2000; Schieber and Santello, 2004; Yu et al., 2010). Such behavior could reflect the saturation of corticospinal projections to individual digits, requiring the recruitment of inappropriate divergent pathways. This could also explain the activation of NCRMs in our task that declined during learning but might perhaps reemerge at stronger contraction levels. Finally, while we here studied hand and forearm muscles, neuroanatomical constraints may be more significant in the control of arm muscles for which the direct corticomotoneuronal projection is weaker. Radhakrishnan et al. (2008) found that subjects preferentially used hand muscles for myoelectric control and were unable to decouple shoulder muscles to optimize their activity. This likely reflects differences in natural upper-limb use; while the task of the arm is often to get the hand to a particular place in space, the hand, once there, can perform a multitude of "abstract" functions from tying a shoelace to playing a piano sonata.
In conclusion, we demonstrate that, during operation of a myoelectric interface, a combination of feedforward and feedback mechanisms contributes to the emergence of new muscle synergies that vary on a trial-to-trial basis. Analysis of intermuscular coherence and perturbation experiments suggest that these represent high-level, cortically mediated processes that progressively optimize motor behavior to abstract task constraints.

\section{References}

Archambault PS, Caminiti R, Battaglia-Mayer A (2009) Cortical mechanisms for online control of hand movement trajectory: the role of the posterior parietal cortex. Cereb Cortex 19:2848-2864.

Audet C, Dennis JE Jr (2003) Analysis of generalized pattern searches. SIAM J Optim 13:889-903.

Berniker M, Jarc A, Bizzi E, Tresch MC (2009) Simplified and effective motor control based on muscle synergies to exploit musculoskeletal dynamics. Proc Natl Acad Sci U S A 106:7601-7606.

Bernstein NA (1967) The co-ordination and regulation of movement. Oxford: Pergamon.

Bizzi E, Giszter SF, Loeb E, Mussa-Ivaldi FA, Saltiel P (1995) Modular organization of motor behavior in the frog's spinal cord. Trends Neurosci 18:442-446.

Bizzi E, Tresch MC, Saltiel P, d'Avella A (2000) New perspectives on spinal motor systems. Nat Rev Neurosci 2:101-108.

Buys EJ, Lemon RN, Mantel GW, Muir RB (1986) Selective facilitation of different hand muscles by single corticospinal neurones in the conscious monkey. J Physiol 381:529-549.

Chhabra M, Jacobs RA (2006) Properties of synergies arising from a theory of optimal motor behavior. Neural Comput 18:2320-2342.

Conway BA, Halliday DM, Farmer SF, Shahani U, Maas P, Weir AI, Rosenberg JR (1995) Synchronization between motor cortex and spinal motoneuronal pool during the performance of a maintained motor task in man. J Physiol 489:917-924.

d'Avella A, Saltiel P, Bizzi E (2003) Combinations of muscle synergies in the construction of a natural motor behavior. Nat Neurosci 6:300-308.

Day BL, Lyon IN (2000) Voluntary modification of automatic arm movements evoked by motion of a visual target. Exp Brain Res 130:159-168.

Diedrichsen J (2007) Optimal task-dependent changes of bimanual feedback control and adaptation. Curr Biol 17:1675-1679.

Diedrichsen J, Shadmehr R, Ivry RB (2010) The coordination of movement: optimal feedback control and beyond. Trends Cogn Sci 14:31-39.

Dietz V, Discher M, Trippel M (1994) Task-dependent modulation of short- and long-latency electromyographic responses in upper limb muscles. Electroencephalogr Clin Neurophysiol 93:49-56.

Dominici N, Ivanenko YP, Cappellini G, d'Avella A, Mondì V, Cicchese M, Fabiano A, Silei T, Di Paolo A, Giannini C, Poppele RE, Lacquaniti F (2011) Locomotor primitives in newborn babies and their development. Science 334:997-999.

Domkin D, Laczko J, Jaric S, Johansson H, Latash ML (2002) Structure of joint variability in bimanual pointing tasks. Exp Brain Res 143:11-23.

Drew T, Kalaska J, Krouchev N (2008) Muscle synergies during locomotion in the cat: a model for motor cortex control. J Physiol 586:1239-1245.

Farmer SF, Bremner FD, Halliday DM, Rosenberg JR, Stephens JA (1993) The frequency content of common synaptic inputs to motoneurones studied during voluntary isometric contraction in man. J Physiol 470:127-155

Fetz EE, Cheney PD (1978) Muscle fields of primate corticomotoneuronal cells. J Physiol (Paris) 74:239-245.

Franklin DW, Wolpert DM (2008) Specificity of reflex adaptation for taskrelevant variability. J Neurosci 28:14165-14175.

Giszter SF, Hart CB, Silfies SP (2010) Spinal cord modularity: evolution, development, and optimization and the possible relevance to low back pain in man. Exp Brain Res 200:283-306.

Hansen NL, Conway BA, Halliday DM, Hansen S, Pyndt HS, BieringSørensen F, Nielsen JB (2005) Reduction of common synaptic drive to ankle dorsiflexor motoneurons during walking in patients with spinal cord lesion. J Neurophysiol 94:934-942.

Harris CM, Wolpert DM (1998) Signal-dependent noise determines motor planning. Nature 394:780-784

Hart CB, Giszter SF (2010) A neural basis for motor primitives in the spinal cord. J Neurosci 30:1322-1336. 
Jackson A, Fetz EE (2011) Interfacing with the computational brain. IEEE Trans Neural Syst Rehabil Eng 19:534-541.

Jackson A, Spinks RL, Freeman TC, Wolpert DM, Lemon RN (2002) Rhythm generation in monkey motor cortex explored using pyramidal tract stimulation. J Physiol 541:685-699.

Jackson A, Gee VJ, Baker SN, Lemon RN (2003) Synchrony between neurons with similar muscle fields in monkey motor cortex. Neuron $38: 115-125$.

Kang N, Shinohara M, Zatsiorsky VM, Latash ML (2004) Learning multifinger synergies: an uncontrolled manifold analysis. Exp Brain Res 157:336-350.

Kilner JM, Salenius S, Baker SN, Jackson A, Hari R, Lemon RN (2003) Taskdependent modulations of cortical oscillatory activity in human subjects during a bimanual precision grip task. Neuroimage 18:67-73.

Knill DC, Bondada A, Chhabra M (2011) Flexible, task-dependent use of sensory feedback to control hand movements. J Neurosci 31:1219-1237.

Krishnamoorthy V, Goodman S, Zatsiorsky V, Latash ML (2003) Muscle synergies during shifts of the center of pressure by standing persons: identification of muscle modes. Biol Cybern 89:152-161.

Kurtzer IL, Pruszynski JA, Scott SH (2008) Long-latency reflexes of the human arm reflect an internal model of limb dynamics. Curr Biol 18:449-453.

Kutch JJ, Kuo AD, Bloch AM, Rymer WZ (2008) Endpoint force fluctuations reveal flexible rather than synergistic patterns of muscle cooperation. J Neurophysiol 100:2455-2471.

Latash ML, Scholz JF, Danion F, Schöner G (2001) Structure of motor variability in marginally redundant multifinger force production tasks. Exp Brain Res 141:153-165.

Latash ML, Scholz JP, Schöner G (2002) Motor control strategies revealed in the structure of motor variability. Exerc Sport Sci Rev 30:26-31.

Lawrence DG, Porter R, Redman SJ (1985) Corticomotoneuronal synapses in the monkey: light microscopic localization upon motoneurons of intrinsic muscles of the hand. J Comp Neurol 232:449-510.

Matthews PB (1991) The human stretch reflex and the motor cortex. Trends Neurosci 14:87-91.

McNemar Q (1947) Note on the sampling error of the difference between correlated proportions or percentages. Psychometrika 12:153-157.

Nadler MA, Harrison LM, Stephens JA (2000) Acquisition of a new motor skill is accompanied by changes in cutaneomuscular reflex responses recorded from finger muscles in man. Exp Brain Res 134:246-254.

Nielsen JB (2002) Motoneuronal drive during human walking. Brain Res Brain Res Rev 40:192-201.

Norton JA, Gorassini MA (2006) Changes in cortically related intermuscu- lar coherence accompanying improvements in locomotor skills in incomplete spinal cord injury. J Neurophysiol 95:2580-2589.

O'Sullivan I, Burdet E, Diedrichsen J (2009) Dissociating variability and effort as determinants of coordination. PLoS Comput Biol 5:e1000345.

Park J, Zatsiorsky VM, Latash ML (2010) Optimality vs. variability; an example of multi-finger redundant tasks. Exp Brain Res 207:119-132.

Radhakrishnan SM, Baker SN, Jackson A (2008) Learning a novel myoelectric-controlled interface task. J Neurophysiol 100:2397-2408.

Rosenberg JR, Amjad AM, Breeze P, Brillinger DR, Halliday DM (1989) The Fourier approach to the identification of functional coupling between neuronal spike trains. Prog Biophys Mol Biol 53:1-31.

Rothwell JC, Traub MM, Marsden CD (1980) Influence of voluntary intent on the human long-latency stretch reflex. Nature 286:496-498.

Schieber MH, Santello M (2004) Hand function: peripheral and central constraints on performance. J Appl Physiol 96:2293-2300.

Scholz JP, Schöner G (1999) The uncontrolled manifold concept: identifying control variables for a functional task. Exp Brain Res 126:289-306.

Scott SH (2004) Optimal feedback control and the neural basis of volitional motor control. Nat Rev Neurosci 5:532-546.

Shinoda Y, Yokota J, Futami T (1981) Divergent projection of individual corticospinal axons to motoneurons of multiple muscles in the monkey. Neurosci Lett 23:7-12.

Takei T, Seki K (2010) Spinal interneurons facilitate coactivation of hand muscles during a precision grip task in monkeys. J Neurosci 30:17041-17050.

Todorov E (2002) Cosine tuning minimizes motor errors. Neural Comput 14:1233-1260.

TodorovE, Ghahramani Z (2004) Analysis of the synergies underlying complex hand manipulation. Conf Proc IEEE Eng Med Biol Soc 6:4637-4640.

Todorov E, Jordan MI (2002) Optimal feedback control as a theory of motor coordination. Nat Neurosci 5:1226-1235.

Todorov E, Li W, Pan X (2005) From task parameters to motor synergies: a hierarchical framework for approximately-optimal control of redundant manipulators. J Robot Syst 22:691-710.

Tresch MC, Jarc A (2009) The case for and against muscle synergies. Curr Opin Neurobiol 19:601-607.

Valero-Cuevas FJ, Venkadesan M, Todorov E (2009) Structured variability of muscle activations supports the minimal intervention principle of motor control. J Neurophysiol 102:59-68.

Yu WS, van Duinen H, Gandevia SC (2010) Limits to the control of the human thumb and fingers in flexion and extension. J Neurophysiol 103:278-289.

Zatsiorsky VM, Li ZM, Latash ML (2000) Enslaving effects in multifinger force production. Exp Brain Res 131:187-195. 